\title{
Core professional and personal values of nurses about nursing in Erbil city hospitals: a profession, not just career
}

\begin{abstract}
Background and objective: Nursing is a caring profession. Caring encompasses empathy for and connection with people. Teaching and role-modeling caring is a nursing curriculum challenge. Caring is best demonstrated by a nurse's ability to embody the five essential to education core values of professional nursing include human dignity, integrity, autonomy, altruism, and social justice. The caring professional nurse integrates these values in clinical practice. Strategies for integrating and teaching core values are outlined and outcomes of value-based nursing education which described to ensure that the legacy of caring behavior by nurses is strengthened for the future nursing workforce
\end{abstract}

Methods: This study aimed to evaluate nurse's values regarding nursing discipline. A cross-sectional study design from $27^{\text {th }}$ of December 2016 to $22^{\text {nd }}$ of January 2017 in Emergency Management Center for Cardiac Surgery and Rizgary Teaching Hospital in Erbil City of Kurdistan Region of Iraq. It consisted of 54 nurses who graduated from the College of Nursing. A non-probability, purposive sample selection was used. Data were collected through the using of a questionnaire by self-report method which consisted of two main parts. First is a sociodemographic characteristic of the nurses and second consisted of 21 items regarding values of nurses about nursing discipline. The data was analyzed through software of SPSS for Windows V.23. It includes descriptive statistical analysis and inferential statistical analysis.

Results: With regards to the age, the highest percentage of the sample $(57.4 \%)$ was between 31 -36 years old, $61.1 \%$ were female, and single $(66.7 \%)$, with $70.4 \%$ had less than 5 years of experience. The items of values of nurses about nursing consisted of 21 items distributed on 9 value domains. Regarding the Human dignity, Social justice, Autonomy, Precision and accuracy in caring, Responsibility, Human relationship, Individual and professional competency, Sympathy, and Trust making domains the means were $80.6 \%, 87.6 \%, 96.3 \%, 98.1 \%, 87 \%, 84 \%, 85.2 \%, 88 \%$, and $84.3 \%$ respectively.

Conclusion: The overall value of nurses regarding the nursing profession is $88 \%$ that is a great result which shows us that most of the nurses have adequate value about their profession.

Keywords: professional values, personal values, nurses, nursing, career
Volume 2 Issue 6 - 2017

Dara Abdulla Al-Banna

Department of Adult Nursing, Hawler Medical University, Iraq

Correspondence: Dara Abdulla Al-Banna, Department of Adult Nursing, Hawler Medical University, Iraq Email daraaq@yahoo.com

Received: February 27, 2017 | Published: May 22, 2017

\section{Introduction}

Nursing is the protection, promotion, and optimization of health and abilities, prevention of illness and injury, facilitation of healing, alleviation of suffering through the diagnosis and treatment of human response, and advocacy in the care of individuals, families, groups, communities, and populations. ${ }^{1}$ According to the International Council of Nurses: "Nursing encompasses autonomous and collaborative care of individuals of all ages, families, groups and communities, sick or well and in all settings. ${ }^{2}$ Nursing includes the promotion of health, prevention of illness, and the care of ill, disabled and dying people. Advocacy, promotion of a safe environment, research, participation in shaping health policy and in patient and health systems management, and education are also key nursing roles". ${ }^{2}$ The nurse is prepared and authorized to engage in the general scope of nursing practice, including the promotion of health, prevention of illness, and care of physically ill, mentally ill, and disabled people of all ages and in all health care and other community settings; to carry out health care teaching; to participate fully as a member of the health care team; to supervise and train nursing and health care auxiliaries; and five to be involved in research. ${ }^{3}$ Carefully integrated values education ensures that the legacy of caring behavior embodied by nurses is strengthened for the future nursing workforce. ${ }^{4}$ Nurses often care for clients whose value systems conflict with their own. Ethics and values which are closely related which both enlightens and complicates the nurse's balancing the ethical principles of the client with those of the health care profession. Nurses must understand their own values in order to practice ethically. ${ }^{5}$ Personal values are values internalized from the society or culture in which one lives. People need societal values to feel accepted, and they need personal values to have a sense of individuality. Professional values are values acquired during socialization into nursing from codes of ethics, nursing experiences, teachers, and peers. ${ }^{6}$ Values something of worth; enduring attitudes about the worth of a person, object, idea, or action. They are important because they influence decisions, actions, even nurse's ethical decision making. A value system is an individual's collection of inner 
beliefs that guides the way the person acts and helps determine the choices the person makes in life. The impact of values on decisions and resultant behaviors is often not considered. Values are similar to the act of breathing; one does not think about them until a problem arises.

\section{Personal values}

Personal values are values internalized from the society or culture in which one lives. People need societal values to feel accepted, and they need personal values to have a sense of individuality.

\section{Professional values}

Professional values are values acquired during socialization into nursing from codes of ethics, nursing experiences, teachers, and peers.

\section{Values clarification}

Values clarification is a process, by which individuals identify, examine and develop their own value. Raths, Harmin, and Simon described a "valuing process":

i. Choosing (cognitive)-beliefs are chosen freely from alternative and reflection and consideration of consequences.

ii. Prizing (affective) - beliefs are prized and cherished.

iii. Acting (behavior) - chosen beliefs are confirmed to others, incorporated into behavior consistently in one's life. ${ }^{4}$

\section{Client values}

To plan effective care, the nurse needs to identify the client's values as they relate to health problems. If the client is unclear or has conflicting values the nurse can help guide the patient to clarify the client's values by using the seven following steps:

List alternatives: Are you considering other courses of action? Tell me about them.

Examine possible consequences of choices: What do you think you will gain from doing that? What benefits do you foresee from doing that?

Choose freely: Did you have any say in that decision? Do you have a choice?

Feel good about the choice: Some people feel good after a decision is made, others feel bad. How do you feel?

Affirm the choice: How will you discuss his with others (family, friends)?

Act on the choice: Will it be difficult to tell your wife about this?

Act with a pattern: How many times have you done that before? Would you act that way again??

and definition of nurses personal and professional core values in health care institutions are important that may affect quality of providing daily care for their clients and there is rare of research regarding nurses personal and professional core values in about in Kurdistan region of Iraq.

\section{Objective}

To evaluate the nurse's values regarding nursing discipline.

\section{Methodology}

\section{Research design}

A cross-sectional.

\section{Duration of the study}

This study was carried out from $27^{\text {th }}$ of December 2016 to $22^{\text {nd }}$ of January 2017.

\section{Setting}

The study was conducted in Emergency Management Center for Cardiac Surgery and Rizgary Teaching Hospital in Erbil City of Kurdistan Region of Iraq.

\section{Study sample}

The population of this study was consisted of 54 nurses who graduated from the College of Nursing. A non-probability, purposive sample selection was used in order to obtain the representative sample according to the following criteria: nurses who have bachelor degree in Nursing Science, agreement to be the subject in the study, both genders, and have good personal communication. Exclusion criteria were administrator nurses and new graduated nurses.

\section{Tools and methods of data collection}

Data were collected through the using of a questionnaire by selfreport method. The questionnaire consisted of two main parts. First part is a sociodemographic characteristic of the nurses which included age, gender, marital status, and years of experience. The second part consisted of 21 items regarding values of nurses about nursing discipline distributed on nine domains of nursing values. The responds contained of three closed ended questions of Always dislike, Depends on situation, and always admire, after correction by researcher the responds converted to 0 for false (incorrect) answers and 1 for True (correct) answers. Data was collected through self-report.

\section{Ethical considerations}

The researcher obtained the permission from the Ethical Committee at the College of Nursing/Hawler Medical University. Before collecting the data, the official permission from the Directorate of Health (Erbil), the administrative of Emergency Management Center for Cardiac Surgery and Rizgary Teaching Hospital was obtained. The researcher promised to keep the participant's information confidential, and use these data for this study only then they explained the purpose of this study to each participant. In addition to above, the researcher told each participant that this is an involuntary work, and they can leave any time even the interview process is not completed.

\section{Statistical analysis}

The data was analyzed through software of SPSS for Windows V.23 (Statistical Package for Science Service) application for statistical data analysis. It includes descriptive statistical analysis (frequency, percentage and mean).

\section{Results}

\section{Socio demographic characteristics of nurses}

Table 1 shows the following characteristics of the study sample regarding the sociodemographic information: With regard to the 
age, the highest percentage of the sample $(57.4 \%)$ was between $31-36$ years old, while the lowest percentage $(42.6 \%)$ was $25-30$ years old. Regarding the gender, highest percentage of the sample $(61.1 \%)$ were female and the lowest were male (38.9\%). It's also shows that the majority were single $(66.7 \%)$, married $(33.4 \%)$, and none were separated, divorced, and widowed. Concerning the years of experience of the sample, the majority of the sample (70.4\%) had less than 5 years while $29.6 \%$ of them had more than and equal to 5 years.

Table I Socio demographic characteristics of nurses

\begin{tabular}{llll}
\hline \multirow{2}{*}{$\begin{array}{l}\text { Socio demographic characteristics of } \\
\text { nurses }\end{array}$} & \multicolumn{2}{l}{$\mathbf{n = 5 4}$} & \\
\cline { 3 - 4 } & & $\mathbf{F}$ & $\%$ \\
\hline Age group (years) & $25-30$ & 23 & 42.6 \\
& $31-36$ & 31 & 57.4 \\
Gender & Male & 21 & 38.9 \\
& Female & 33 & 61.1 \\
& Single & 36 & 66.7 \\
Marital status & Married & 18 & 33.4 \\
& Separated & 0 & 0 \\
& Divorced & 0 & 0 \\
Years of experience & Widowed & 0 & 0 \\
& $<5$ & 38 & 70.4 \\
\hline
\end{tabular}

\section{Values of nurses about nursing}

Table 2 shows the items of values of nurses about nursing which consist of 21 items distributed on 9 value domains. Regarding the

Table 2 Values of nurses about nursing.
Human dignity domain that consists of 4 items about patients respect, avoid punishing, and cultural diversity, all of the study samples answered correctly in a high percentage (mean $80.6 \%$ ). Concerning the Social justice that have 3 items of equality in distributing health care services equally between patients $87.6 \%$ of the study samples were correctly answered. The item related to Autonomy in decision making domain, $96.3 \%$ of the nurses were correctly responded in this item which focused on the independency value in nursing in decision making. About the Precision and accuracy in caring domain, the three items were stressed on forgiving, kindness, and helping to feel security and safety values had great corrected answers (98.1\%). Regarding Responsibility domain that asked a question about relationship between salary and duties, the item studying hard to get good salary and position, is one of the nurse's duties $87 \%$ of the nurses were correctly responded. Concerning the Human relationship domain which has 3 items that focused on teaching/learning process involvement and telling truth for patient's family, $84 \%$ were answered correctly. The item related to Individual and professional competency that include 2 items of nurse's knowledge and skill development with up-to-date information in a competence level, $85.2 \%$ of the study sample were answered correctly. About the domain of Sympathy value of nurses in nursing discipline which stressed on the self- controlling and not express anger values, most of the nurses were answers correctly in a high percentage of $88 \%$. The final domain in the present study related to the making a bridge of trust between the patient- family- health care providers, all of the nurses they know the value of importance in trust making, but only $84.3 \%$ of them they had information about telling a lie, that may he/she makes the situation more comfortable for the clients which some of them they answered depends on the situation respond. So, as a general the overall value of nurses regarding the nursing profession is $88 \%$ that is a great result which shows us that most of the nurses have adequate value about their profession.

\section{Items of values of nurses about nursing $(n=12)$}

\begin{tabular}{ll} 
Not correct & Correct \\
\hline F $\%$ & F $\%$
\end{tabular}

\section{Human dignity}

To respect a client's personality, the nurse should explains nursing care and procedures before starting the care

For unethical behavior by the clients, it's necessary for the nurse to punish them at the first time.

It's not necessary to care for end of life patients

$\begin{array}{llll}7 & 13 & 4787\end{array}$

When the nurse put a plan for nursing care of a client with a different cultural, he/she should identify how these cultural variables affect the health problem.

\section{Social justice}

The nurse should proud when he/she provide nursing care for very important persons (VIP)

It is necessary for the nurse to know the client's social position during nursing care

The nurse should differentiate between the rich and the poor clients during providing the health care

$0 \quad 54 \quad 100$

\section{Autonomy in decision making}

The nurse should being independent, original, non-conformist, and different from the other specialties

\section{Precision and accuracy in caring}

The nurse should not forgiving clients when they harm during providing nursing care

The nurse should being kind to patient, even if they do things contrary to one's own beliefs 
Table Continued....

\section{Items of values of nurses about nursing $(n=\mid 2)$}

Responsibility

Studying hard to get good salary and position, is one of the nurse's duties

4787

\section{Human relationship}

During the nursing teaching/learning, the nurse must not encourage patient's relative to attend this process

The nurse should not always telling the truth, because it may hurt the patient or others

Its important for the nurse to notify all of the patent's relative about the his/her health problem

\section{Individual and professional competency}

The nurse should develop new and different ways of doing holistic nursing care

It's a nurse's obligation to studying constantly in order to become a well-educated nurse

\section{Sympathy}

The nurse should not express anger, even when he/she has a reason for doing it

In the nursing care, practicing self-control is an essential value to build a good personality

Trust

The nurse should telling a lie, may he/she makes the situation more comfortable for the clients

It's important to make a trust between the nurse and the client before starting any nursing care

\section{Discussion}

The present study aimed to evaluate the nurse's values regarding the nursing discipline among nurses who had bachelor degree. The findings reveled that most of the study sample were in the age group between 31-36 years old, female, single, with the years of experience of more than and equal to 5 years in nursing care. This findings supported by the study which was done by Shih et al. ${ }^{8}$ in 2008 under the title Perspectives on professional values among nurses in Taiwan which they found the same results. ${ }^{8}$ Concerning the nurse's values about nursing which contained 9 value domains of Human dignity, Social justice, Autonomy in decision making, Precision and accuracy in caring, Responsibility, Human relationship, Individual and professional competency, Sympathy, and Trust making, most of the nurses have very good information regarding these values in high rate. All of the findings in the present study were agreed by many studies which were done in different countries in a different time. The study which was done in 2011 in Iran under the title Perceived ethical values by Iranian nurses by Shahriari et al. ${ }^{9}$ showed that the Iranian nurses recognized the observance of ethical values essential to patient care, such as maintaining patient privacy, practice integrity, professional commitment, human relationships, justice, honesty and the promotion of professional competence. ${ }^{9}$ Additionally, in a study which was done by Konishi et al. ${ }^{10}$ in 2009 under the title The Japanese Value of Harmony and Nursing Ethics, they found that the most important virtue for Japanese nurses in their studies who practice harmony was politeness, expressed by respect for the others, by touching the hearts of the people concerned, the nurses created the outcome of goodness, peace and growth of all members in the place, not limited to 'the patient good'. ${ }^{10}$ It's noteworthy that the study which was done in Turkey under the title Burnout and Nurses' Personal and Professional Values by Altun ${ }^{11}$ he concluded that the nurses' personal and professional values play an important role in the degree of burnout they experience. Equality, altruism and aesthetics were ranked first by those experiencing high levels of emotional exhaustion, and freedom was a priority value for those with a low degree of emotional exhaustion, also the author's study showed that Freedom, altruism and truth were ranked first by those with prominent feelings of personal accomplishment, and equality and aesthetics were priority values for those with less feeling of personal accomplishment. All nurses, therefore, need to identify and clarify their own personal values, beliefs and assumptions about basic truths. ${ }^{11}$ Moreover, in a study which was done by Jormsri et al. ${ }^{12}$ in Thailand, reveled that the process of moral competence is formed and based on the Thai nursing value system, including personal, social and professional values; eight attributes as indicators of moral competence are identified and selected from three basic values. The eight attributes are kindness, compassion, sympathetic, equanimity, responsibility, discipline, honesty, and respect for human values, dignity and rights, all model of moral competence is presented in nursing practice in Thailand. ${ }^{12}$ Furthermore, in a study which was done in China under the title Nursing values in China: The expectations of registered nurses by Pang et al. ${ }^{13}$ in a qualitative approach to obtain thick descriptions of nurses' perceptions and experiences of professional values, they found that nearly all the participants assented to ethical and legal responsibility and accountability for their remarks and practices as the basic requirement, and all the participants stated the Chinese term " shen du" which means to be self-disciplined and to behave ethically, even when not being supervised. Nearly all the participants mentioned that nurses should take measures to shield patients' intimate parts from being exposed to others when providing care. Most of the participants stressed the importance of updating nurses' "intellectual capital" by lifelong learning. They said that nurses should be in line with the development of the profession, including their thinking, vision, and professional knowledge and skills. ${ }^{13}$ In the above study Pang et al. ${ }^{13}$ showed that the eight nurse educators and one clinical 
nurse emphasized participation in research in order to contribute to evidence- based professional knowledge. Some talked about improving intra disciplinary and interdisciplinary cooperation and team spirit in nurses' work. Nearly all the participants expressed a belief in delivering respectful service to patients of diverse creeds, cultures, genders, and economic status. In this study, the participants reached a consensus on protecting patients' privacy and confidentiality, as well as respecting them. With regard to justice, nearly all the participants mentioned providing equal services to patients irrespective of their background. ${ }^{11,13}$ Finally, a study which was done in Israel by Rassin in 2008 under the title Nurses' Professional and Personal Values among 323 Israeli nurses, who were asked about 36 personal values and 20 professional values., the author showed that the three fundamental professional nursing values of human dignity, equality among patients, and prevention of suffering, were rated first and for personal values, honesty, responsibility and intelligence were rated first. Nurses with a bachelor's degree valued nursing research higher than did all other groups. ${ }^{14}$

\section{Conclusion}

The present study concluded that all nurses who participated in this study were graduated from the college of nursing with bachelor degree, had adequate information regarding nursing values, and it's very important because these values directly affecting the health care services which the nurses are the core in providing health care for all patients.

\section{Recommendations}

For the schools of nursing: it's preferable to put philosophy of nursing in the curriculum of nursing lectures for students to have a basic adequate knowledge regarding the values in nursing discipline. For institutions that provide nursing health care services: All nurses should get enough information regarding nursing values through courses or workshops to improve quality of health care services.

\section{Acknowledgements}

None.

\section{Conflict of interest}

The author declares no conflict of interest.

\section{References}

1. American nurse association. What is Nursing? Georgia: USA; 2016.

2. Crosta MP. What is nursing? What does a nurse do? Publications of the MediLexicon International Ltd. UK: Springer; 2015.

3. International council of nursing. Definition of nursing, Switzerland: Publications of the ICN; 2015.

4. Fahrenwald NL, Bassett SD, Tschetter L, et al. Teaching Core Nursing Values. Journal of Professional nursing. 2004;21(1):46-51.

5. Dewit S. Fundamental concepts and skills for nursing. USA: WB Saunders Company; 2001. p. 30-45.

6. Kozier B, Erb GL, Berman A, et al. Fundamentals of Nursing. 3rd ed. Australia: Pearson Australia; 2014.

7. Amsale C, Ato M, Tsehay S. Introduction to Professional Nursing and Ethics. Ethiopia Public Health Training Initiative, Addis Ababa University, Ethiopia; 2006. p. 25-31.

8. Shih FJ, Lin YS, Smith MC, et al. Perspectives on professional values among nurses in Taiwan. J Clin Nurs. 2009;18(10):1480-1489.

9. Shahriari M, Mohammadi E, Abbaszadeh A, et al. Perceived ethical values by Iranian nurses. Nurs Ethics. 2011;19(1):30-44.

10. Konishi E, Yahiro M, Nakajima N, et al. The Japanese value of harmony and nursing ethics. Nurs Ethics. 2009;16(5):625-636.

11. Altun I. Burnout and nurses personal and professional values. Nurs Ethics. 2002;9(3):270-278.

12. Jormsri P, Kunaviktikul W, Ketefian S, et al. Moral competence in nursing practice. Nurs Ethics. 2005;12(6):582-594.

13. Pang D, Senaratana W, Kunaviktikul W, et al. Nursing values in China: The expectations of registered nurses. Nurs Health Sci. 2009;11(3):312317.

14. Rassin M. Nurses' professional and personal values. Nurs Ethics. 2008;15(5):614-630. 